\title{
Experiments at the W.M. Keck Observatory to support the Thirty Meter Telescope design work
}

Warren Skidmore, Tony Travouillon, Reed Riddle, Kyle Kinoshita, Richard Johnston, et al.

Warren Skidmore, Tony Travouillon, Reed Riddle, Kyle Kinoshita, Richard Johnston, Phil Murg, Hugh Thompson, Richard Matsuda, Mark Colavita, Grant Tolleth, Robert Goodrich, Elizabeth Chock, Hilton Lewis, Sergey Panteleev, "Experiments at the W.M. Keck Observatory to support the Thirty Meter Telescope design work," Proc. SPIE 7733, Ground-based and Airborne Telescopes III, 773350 (6 August 2010); doi: 10.1117/12.857960

Event: SPIE Astronomical Telescopes + Instrumentation, 2010, San Diego, California, United States 


\title{
Experiments at the W.M. Keck Observatory to support the Thirty Meter Telescope Design Work
}

\author{
Warren Skidmore $^{a}$, Tony Travouillon ${ }^{a}$, Reed Riddle ${ }^{b}$, Kyle Kinoshita ${ }^{c}$, Richard Johnston ${ }^{c}$, \\ Phil Murg $^{a}$, Hugh Thompson ${ }^{a}$, Richard Matsuda ${ }^{c}$, Mark Colavita ${ }^{d}$, Grant Tolleth ${ }^{c}$, Robert \\ Goodrich $^{c}$, Elizabeth Chock ${ }^{c}$, Hilton Lewis ${ }^{c}$, and Sergey Panteleev ${ }^{c}$, \\ ${ }^{a}$ TMT Observatory Corporation, 2632 E. Washington Blvd., Pasadena, CA 91107, USA; \\ ${ }^{b}$ Caltech Optical Observatories, MS 105-24, California Institute of Technology, Pasadena, CA \\ 91125, USA; \\ ${ }^{c}$ W.M. Keck Observatory, 65-1120 Mamalahoa Highway, Kamuela, HI 96743, USA; \\ ${ }^{d}$ Jet Propulsion Laboratory, California Institute of Technology, Pasadena, CA 91109, USA
}

\begin{abstract}
In order to validate various assumptions about the operating environment of the Thirty Meter Telescope (TMT), to validate the modeling packages being used to guide the design work for the TMT and to directly investigate the expected operation of several subsystems we have embarked on an extensive campaign of environmental measurements at the Keck telescopes. We have measured and characterized the vibration environment around the observatory floor and at certain locations on the telescope over a range of operating conditions. Similarly the acoustic environment around the telescope and primary mirror has been characterized for frequencies above $2 \mathrm{~Hz}$. The internal and external wind and temperature fields are being measured using combined sonic anemometer and PRT sensors. We are measuring the telescope position error and drive torque signals in order to investigate the wind induced telescope motions. A scintillometer mounted on the telescope is measuring the optical turbulence inside the telescope tube. This experimental work is supplemented by an extensive analysis of telescope and engineering sensor log files and measurements, primarily those of accelerometers located on the main telescope optics, primary mirror segment edge sensor error signals (residuals), telescope structure temperature measurements and the telescope status information.
\end{abstract}

Keywords: dome seeing, computational fluid dynamics, control system, edge sensors, humidity, vibration, actuators

\section{INTRODUCTION}

The design of the TMT is almost wholly based on models. Specifically, four different types of modeling tools are being used in various combinations, Computational Fluid Dynamics (CFD), ${ }^{1}$ Finite Element Analysis (FEA), ${ }^{2}$ structural thermal models ${ }^{3}$ and models of the control systems. ${ }^{4,5}$ By driving these models with a well developed Disturbance Model the overall effect on the Normalized Point Source Sensitivity (PSSN) can be calculated and thus within the model the design of the systems can be tuned to maximize the PSSN.

Given the need for high confidence in the modeling tools, we have embarked on a model validation effort at the W.M. Keck Observatory. We are precisely measuring the environment, environmental disturbances and the response of some of the telescope systems in order to provide inputs or boundary conditions to models of the Keck telescopes. This produces an extremely representative Disturbance Model and allows a comparison between the predicted and actual telescope response or the predicted and actual dome seeing, comparing the predictions of the models against real measurements. Thus we are anchoring the design of TMT into existing observatories.

Additionally, many of the subsystems that will operate on TMT are very similar to those on the Keck Telescopes. Critically the Keck Primary Control System uses segment edge sensors, actuators and a segment support system that is very similar to that planned for use on TMT. By data mining the telescope engineering sensor and log files we can directly investigate the performance of these components on the Keck telescopes and gain important guidance for the design of the TMT subsystems.

Send correspondence to Warren Skidmore: e-mail: was@tmt.org, Telephone: +1-626-395-6956

Ground-based and Airborne Telescopes III, edited by Larry M. Stepp, Roberto Gilmozzi, Helen J. Hall

Proc. of SPIE Vol. 7733, 773350 - (c) 2010 SPIE · CCC code: 0277-786X/10/\$18 · doi: 10.1117/12.857960 

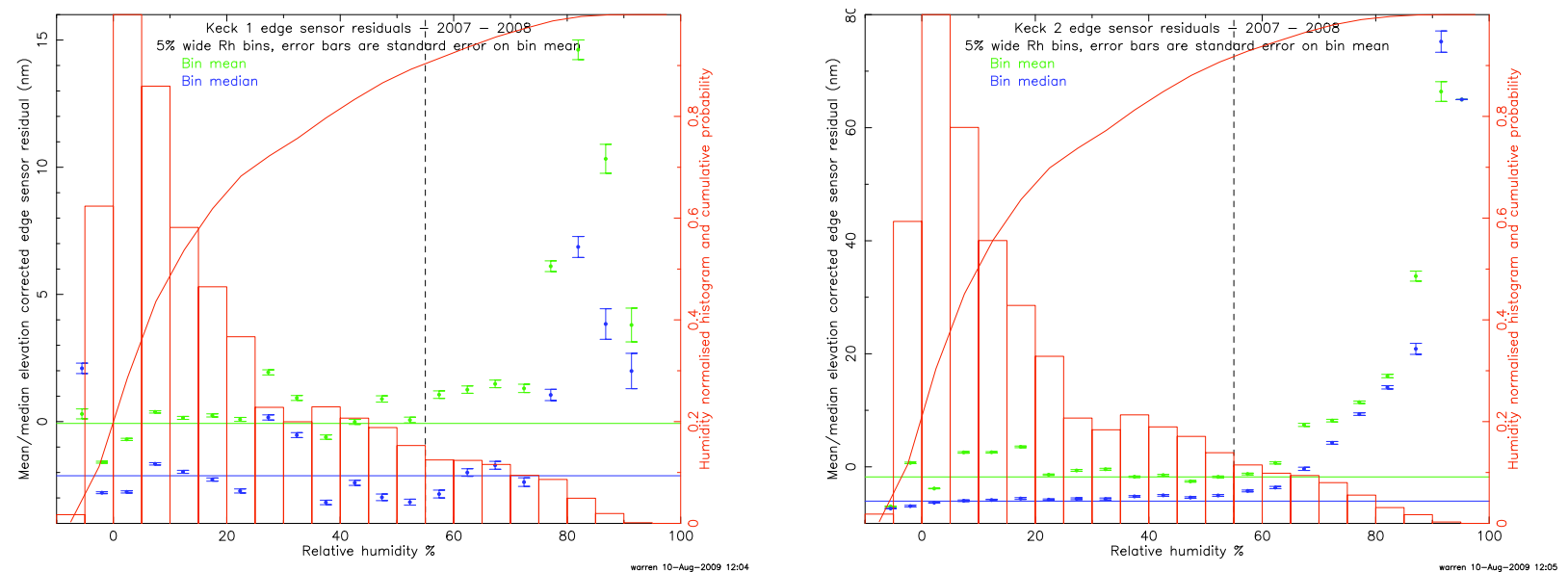

Figure 1. Left: The Keck 1 segment edge sensor residual as a function of humidity. This includes only 'dome open' measurements when the telescope is not parked. Also plotted in red is the relative humidity probability histogram and cumulative probability curve which has been included in the TMT disturbance model. Right: The Keck 2 segment edge sensor residual as a function of humidity. The larger response of the Keck 2 edge sensors is clearly seen.

\section{DATA MINING EFFORTS}

\subsection{Keck telescope log files}

Log files used in data mining studies include measurements of the primary mirror segment edge sensor residuals and accelerometer measurements from the optics mounted accelerometers that are used with the interferometer system. ${ }^{6}$ Data contained in the log files are routinely captured and cover long temporal baselines, typically several years. Also used are telescope status log files for determining particular telescope states (i.e. parked, slewing, observing a science target, etc.).

\subsection{Primary mirror alignment and control system (M1 ACS) edge sensor response to humidity}

At a particular elevation angle, for each primary mirror segment edge sensor there is a desired edge sensor reading (taken from a previously determined look up table). The residual for a sensor is the difference between the desired and actual reading, the residuals are minimized by the M1 ACS by pistoning the M1 segment actuators, which pistons, tips and tilts the segments. There are 102 actuators ( 3 per segment) and 168 edge sensors so the residual cannot be reduced to zero. The residuals are sampled at $100 \mathrm{~Hz}$, passed through a $0.2 \mathrm{~Hz}$ low pass double pole filter and the $2 \mathrm{~s}$ average of the filter output is recorded every $60 \mathrm{~s}$ in the ACS edge sensor residual log file along with the telescope altitude, primary mirror temperature and azimuth and the humidity on the Keck 1 dome.

Measurements are available from 20060208 until 20090902 for Keck 1 (the date of the analysis) and 2008 1002 for Keck 2 (the date of the last ACS log file). During this time there were no segment exchanges on Keck 2 but several on Keck 1 in the first few months of the period. During this period 1st generation sensors were the majority of sensors on Keck 2 and 2nd generation sensors were the majority on Keck 1.

It was found that the edge sensor residuals increased during high humidity periods, this effect was small for Keck 1, 5nm to $10 \mathrm{~nm}$ increased RMS residual above $75 \%$ Rh but much larger for Keck 2, upto 70nm increased RMS residual rising from $65 \% \mathrm{Rh}$ (see Figure 1. We can conclude that this response is partially due to the sensor build quality. There are reports that the build quality of the 2nd generation of sensors constructed was much more satisfactory than for the first generation of sensors. There was no clear indication of systematic changes in the Keck 1 edge sensor humidity response as segments were exchanged. No connection was found between the edge sensor humidity response and the occurrence of M1 alignment runs, instrument changes or M1 ACS reset operations. A method to calibrate the individual edge sensor humidity response was tested and it is expected that the effect of high humidity on the edge sensor residuals can be reduced by about $40 \%$. 


\subsection{Vibrations of primary mirror segments (+ other optical elements)}

A set of $151 \mathrm{D}$ accelerometers are located around the optical components of each of the Keck telescopes. ${ }^{6}$ The axis of each sensor is aligned along the optical path and the sensor measurements used to correct for path length differences during interferometer observations. Accelerometer measurements are made at $1 \mathrm{kHz}$ and $10 \mathrm{~s}$ samples are recorded every 20 minutes. These 10 s samples are cross referenced against telescope state.

For TMT the most relevant measurements are those on the M1 segments, measurements from 6 sensors in radial pairs on the inner and outer edges of three second ring segments were studied in detail. The power spectra of all sensors in all 10s data sets were calculated. This allowed a statistical and temporal analysis of the power at each frequency, identification of frequencies of interest and correlation of vibrations against telescope state.

The dominant vibrational frequency was close to the $30 \mathrm{~Hz}$ expected from 4 pole motors. The mean amplitude around the $29.4 \mathrm{~Hz}$ peak was $26 \mathrm{~nm}$ of M1 surface motion above six sensors. However the amplitude varied between $5 \mathrm{~nm}$ and $75 \mathrm{~nm}$. Temporal variations of the $29.4 \mathrm{~Hz}$ oscillation are seen on all time scales and do not show any regular pattern. Other periodicities present include 54, 58, 60, 243 and $400 \mathrm{~Hz}$.

\subsection{Transmission of vibration through primary mirror actuators}

To measure the transmission of the M1 cell vibrations through the actuators, the segment support structure and into the M1 segments, two of the M1 segment accelerometers were temporarily relocated from the radially inner edge of their particular segment to the M1 cell structure directly beneath the 2nd sensor on that segment. The accelerometer DAS was allowed to run as normal (see Section 2.3). 19 days of measurements were gathered. This enabled the determination of the ratio as a function of frequency of the M1 cell and segment vibration power spectra. Also investigated was the coherence as a function of frequency between the cell and segments, between different parts of the cell, between either edge of a segment and between segments.

Power spectra of nighttime measurements of the M1 cell and segment vibrations show that the $29 \mathrm{~Hz}$ vibration dominates. Below $24 \mathrm{~Hz}$ the vibration transmission from the M1 cell to the mirror has a gain of 1, between 24 $\mathrm{Hz}$ and $25 \mathrm{~Hz}$ the gain rises to 2 and is 2 above $30 \mathrm{~Hz}$. The mirror cell mean vibration amplitude at $29 \mathrm{~Hz}$ is 13 $\mathrm{nm}$. Tip/tilt and piston resonances of the segment support assembly are seen at 35 and $43 \mathrm{~Hz}$ respectively.

\subsection{Phasing of $30 \mathrm{~Hz}$ vibrations of the primary mirror segments from AO telemetry}

The Keck NGS AO system measures the wave front at $1054 \mathrm{~Hz}$ with $56 \mathrm{~cm}$ spacing on M1 (segment size is $1.56 \mathrm{~m}$ face to face, $1.8 \mathrm{~m}$ corner to corner), the overall AO servo bandwidth is $100 \mathrm{~Hz}$. The AO telemetry consists of the DM piston signals and allows an analysis of periodic motions of the whole of the M1 surface. We know from accelerometer measurements that the segments vibrations around $29 \mathrm{~Hz}$ to $30 \mathrm{~Hz}$ are the most problematic (see Section 2.3), and this frequency range was analyzed in detail in a $126.2 \mathrm{~s}$ long telemetry data set.

$29 \mathrm{~Hz}$ vibrations appear correlated on spatial scales of about $1.3 \mathrm{~m}$. The spatial distribution of vibration amplitude is consistent with that measured using accelerometers.

\section{EXPERIMENTAL EFFORTS}

\subsection{Measurements to validate design models}

Experiments are being run to gather measurements used for validation of design models and defining the TMT disturbance model. The experimental work has necessitated the installation of new equipment onto the telescope. All measurements being collected are cross referenced against telescope status, i.e. the telescope position, speed and whether science observations are being conducted. Meteorological parameters from the CFHT weather tower were also collected and experimental measurements labelled for particular external wind speed and relative direction between telescope and external wind direction. 


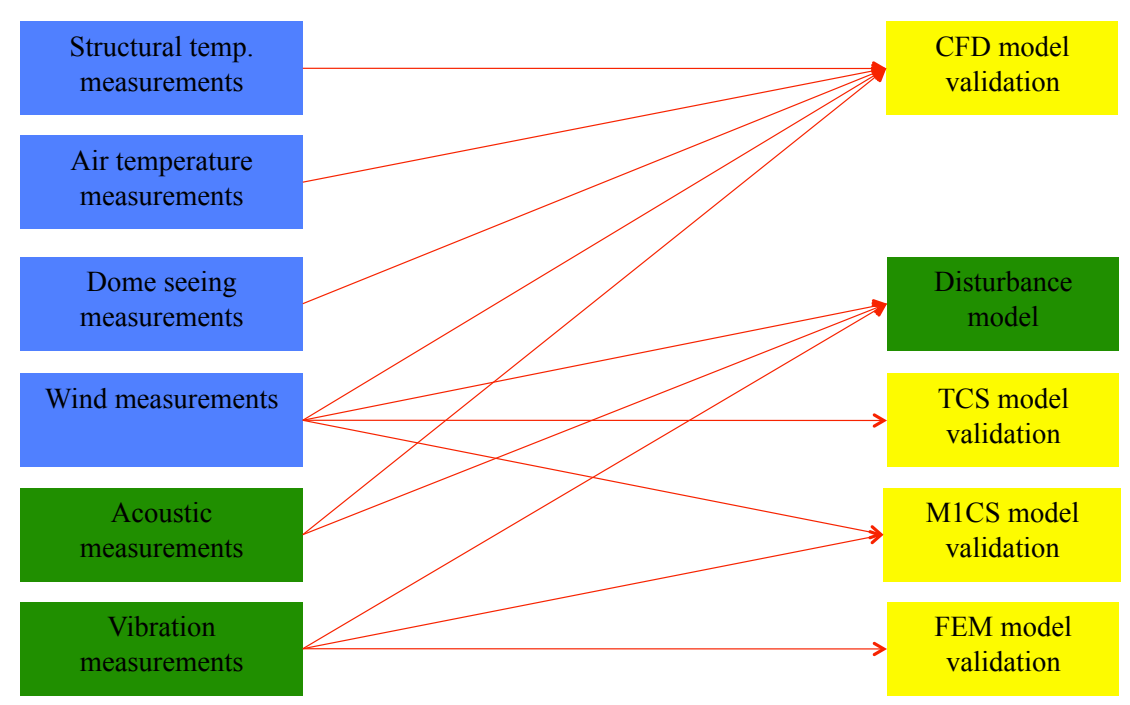

Figure 2. Figure showing how the experimental measurements feed into the various studies.

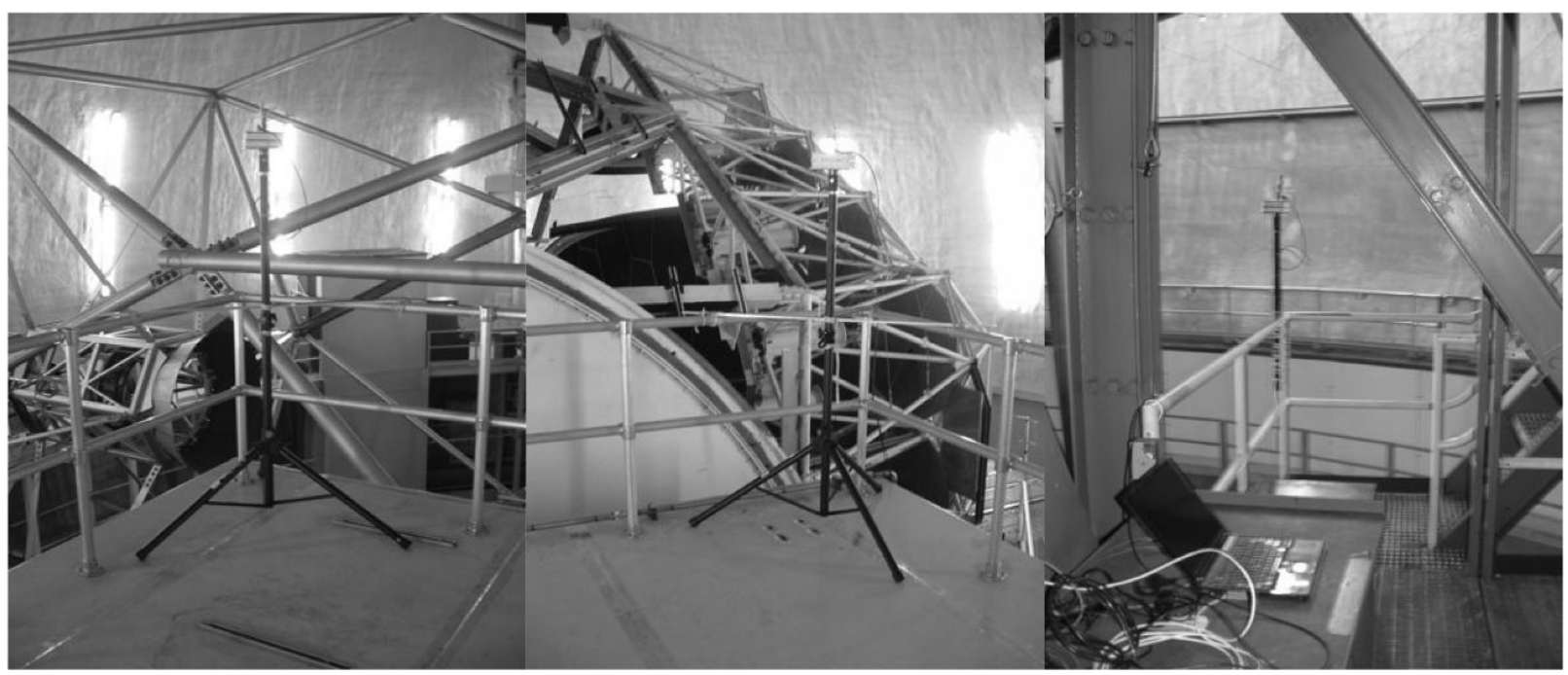

Figure 3. Acoustic sensors at the Keck 1 telescope, left and middle are views of the top of the AO enclosure, right is down below the elevation axis to the rear of the telescope. During science observations two sensors were in front of the mirror plane, one sensor was behind. 


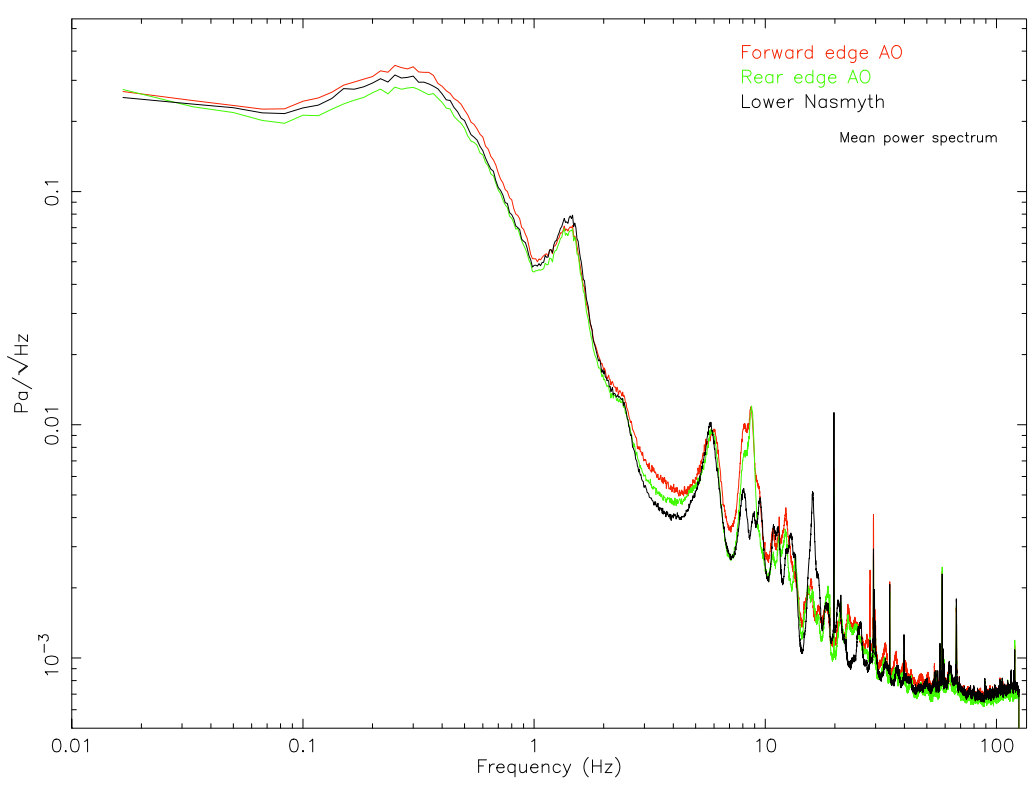

Figure 4. The mean power spectra of the acoustic signals from the three sensors shown in Figure 3.

\subsection{Acoustic and vibration measurements}

The acoustic environment of an observatory has not previously been quantified. Acoustic disturbances can induce vibrations of the telescope systems and cause disturbances of the M1 surface due front-back pressure differences. Acoustic disturbances have to be mitigated and/or rejected by the telescope systems. For TMT the largest concern is acoustic coupling to the M1 segments, both at vibrational frequencies (tens of $\mathrm{Hz}$ ) and sub-audible $(\leq 5 \mathrm{~Hz})$ sounds resulting from shear layer or Helmholtz resonances of the air above the enclosure aperture.

Figure 3 shows acoustic sensors set up on the left side of the Keck 1 telescope. The acoustic sensors and amplifiers had a $3 \mathrm{~dB}$ point at just greater than $1 \mathrm{~Hz}$ and were sensitive down to about $0.5 \mathrm{~Hz}$. They were mounted on vibration isolation units. The acoustic sensors were run for 9 days continuously at sampling frequencies of $250 \mathrm{~Hz}$ (days 1 to 5 ) and $1 \mathrm{kHz}$ (days 6 to 9).

Figure 4 shows the mean acoustic pressure PSDs from times when the telescope was observing science targets. The median RMS pressure for all sensors over all frequencies above $0.9 \mathrm{~Hz}$ was $0.01 \mathrm{~Pa}$ and around the $29.4 \mathrm{~Hz}$ peak vibration frequency was $0.0012 \mathrm{~Pa}$. This level of acoustic pressure close to $30 \mathrm{~Hz}$ would cause a displacement of the TMT segments of $1 \times 10^{-11} \mathrm{~m}$ so is not a significant problem. No evidence for acoustic shear layer resonance above the Keck dome opening was found however all frequency bands showed an increase in RMS amplitude with increasing wind speed.

Vibrational disturbances are nowadays a well known problem at observatories with adaptive optics systems. For large aperture telescopes and high contrast AO systems the vibration environment limits the system performance. TMT uses the Keck vibrational environment as a benchmark.

Figure 5 shows the $3 \mathrm{D}$ accelerometer sensor used during a campaign at Keck 1, simultaneous with the acoustic measurements. Accelerometer measurements were made at several locations around the pier and pintle bearing, the sensors being firmly bolted to the pier or the telescope foundations. Measurements were also made on the structural members of the left Keck 1 Nasmyth platform. At each location, measurements were made for two to three days so that significant numbers of measurements were collected during telescope science operations.

Vibrations of the telescope pier and Nasmyth platform were characterized. Between $4 \mathrm{~Hz}$ and $50 \mathrm{~Hz}$ the RMS integrated amplitude is typically $\sim 10 \mathrm{~nm}$ in the vertical and horizontal directions for all locations during science observations. For $29.4 \mathrm{~Hz} \pm 1.5 \mathrm{~Hz}$ the RMS amplitude around the pier is typically $\sim 1 \mathrm{~nm}$ vertically and 


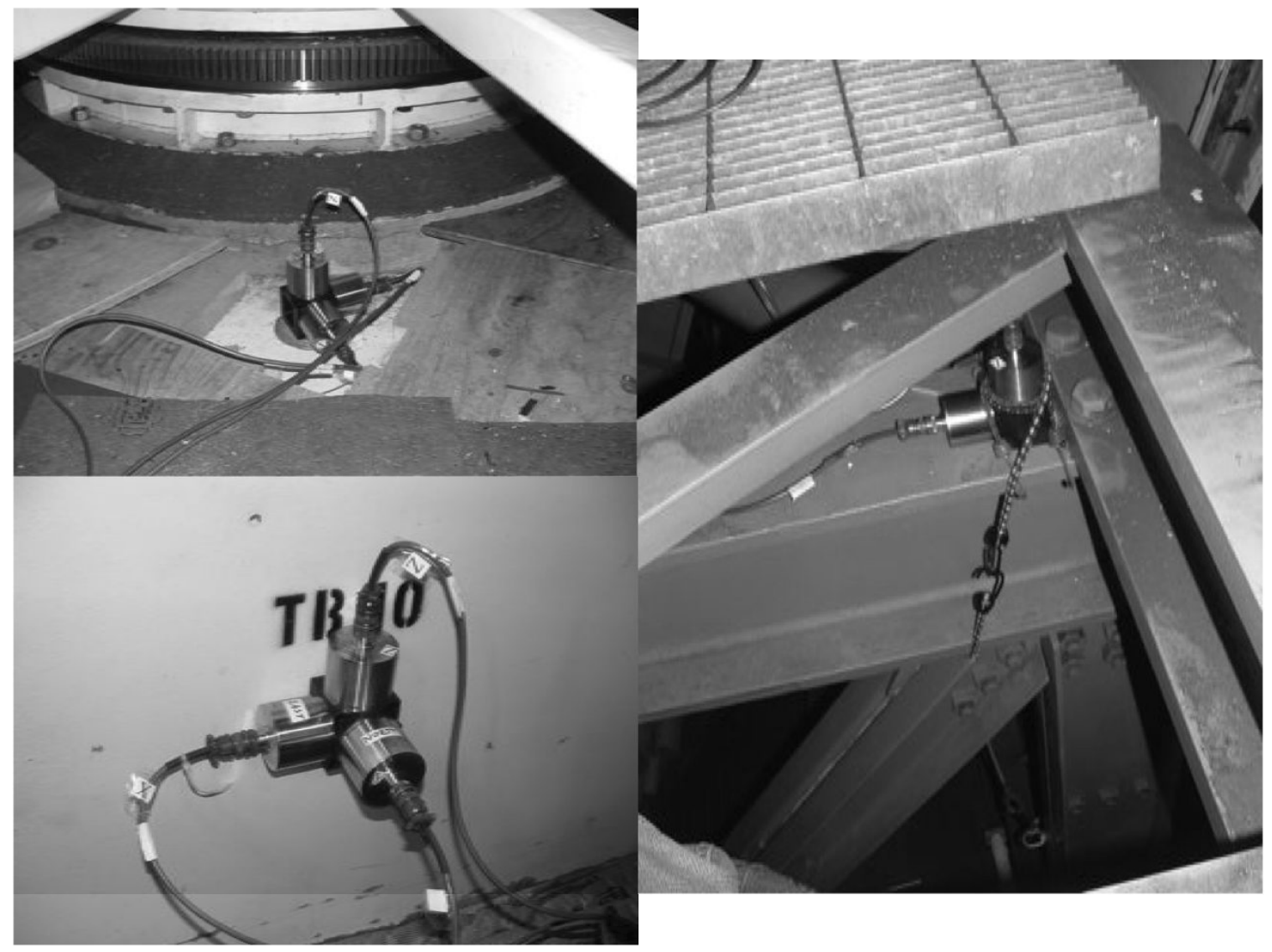

Figure 5. Views of the 3D accelerometer sensor on the Nasmyth platform supports, on the pier and next to the pintle bearing.

$\sim 0.7 \mathrm{~nm}$ horizontally. The vibrations on the Nasmyth structure are $\sim 2 \mathrm{~nm}$ vertically and parallel to the elevation axis and $\sim 0.4 \mathrm{~nm}$ in the horizontal direction perpendicular to the elevation axis.

There is a clear bimodal distribution of $30 \mathrm{~Hz}$ vibration amplitudes, indicating the presence of frequently operated 4 pole motors. The $29.4 \mathrm{~Hz}$ vibration amplitude varies systematically between 0.1 and $2.1 \mathrm{~nm}$ in all axes, generally higher during science observations. No definite evidence of wind induced seismic pier motions was found, the RMS displacement between 0 to $4 \mathrm{~Hz}$ has no significant correlation with external wind speed.

\subsection{Wind and air temperature field}

Wind flow over and around the telescope causes wind induced disturbances that need to be rejected by the telescope control system. Air moving over and through the observatory thermally interacts with the observatory structures. Air temperature fluctuations and motions of the air result in optical turbulence. ${ }^{7}$

We are investigating the effect of wind on the telescope structure motions and the control system's ability to mitigate those effects. We are also seeking to validate the Computational Fluid Dynamics (CFD) models being used for the TMT design by making predictions of the conditions at the Keck telescopes, the wind and temperature fields are inputs to those models.

Figure 6 shows the locations of sonic anemometer and air temperature combined probes on the Keck 2 telescope structure. Three sonic anemometer/air temperature sensors were installed in July 2009 on the M2 ring. The 4th was installed in Februry 2010 in the M1 cell. A fifth combined probe was set up on the top of the Keck 2 dome in may 2010 (see left of Figure 7). The sonic anemometers produce a 3D wind vector at $32 \mathrm{~Hz}$, the 


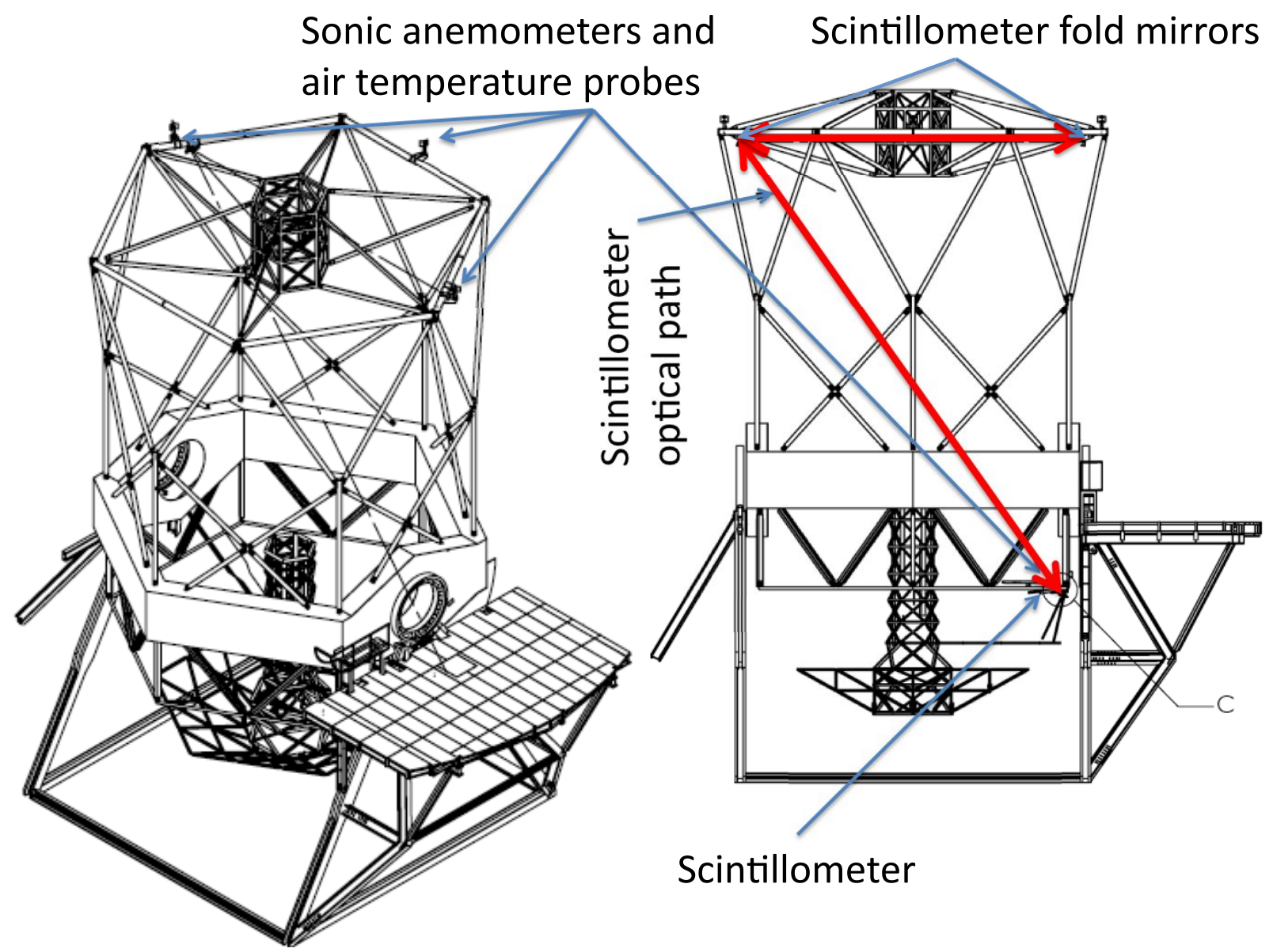

Figure 6. Sensor locations on the Keck 2 telescope. Fold mirrors for the scintillometer laser are located on the M2 ring. Peak scintillometer sensitivity is around the M2 region. 


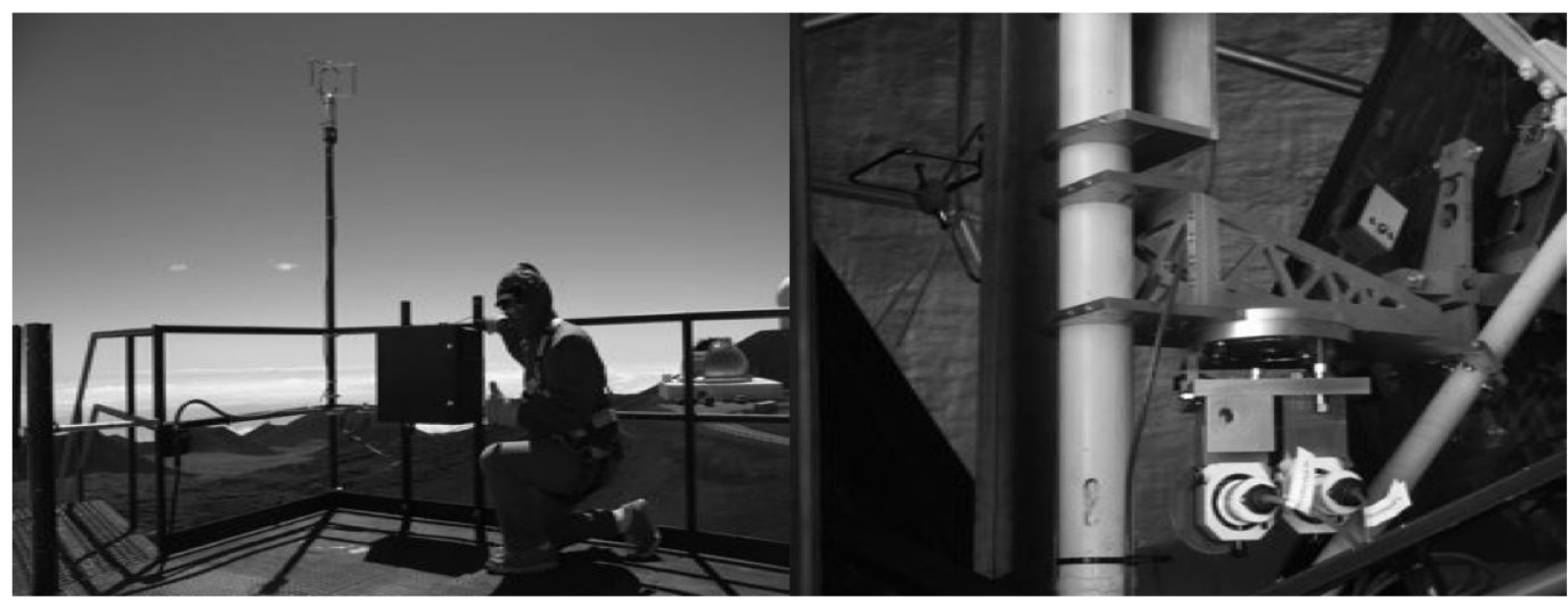

Figure 7. Left: Sonic anemometer and temperature sensor installed on Keck 2 dome top. Right: Scintillometer transmitter and receiver installed alongside the Keck 2 primary mirror.

temperature probes are PRTs read at $10 \mathrm{~Hz}$. Data is collected continuously. Telescope structural temperatures are gathered every 60 s by the Keck engineering sensors system.

In addition to air and structural temperatures we are planning to measure the temperatures of and heat fluxes from equipment inside the Keck 2 dome. Heat and cold plumes contribute to the thermal turbulence and thermal radiation affects the equilibrium of surrounding structures.

\subsection{Optical turbulence (dome seeing)}

CFD models are being used to predict the optical turbulence field inside the Keck 2 dome. Predictions indicate that the highest turbulence is in the region between the dome aperture and telescope top end.

To measure optical turbulence inside the dome in February 2010 we installed a laser scintillometer onto the telescope, see Figure 6 and the right of Figure 7. The laser transmitter and receiver are co-located at the left side of the primary mirror. The laser path goes diagonally across the telescope tube to the M2 ring, hits an angled fold mirror, goes directly across the M2 region to the opposite side of the M2 ring, hits a second fold mirror which sends the beam straight back. Due to telescope tube and equipment flexure the scintillometer only produces valid turbulence measurements when the telescope elevation is between $48^{\circ}$ and $89^{\circ}$. We have assessed the impact of the scintillometer laser light on science operations involving the NIRSPEC instrument and MAGIQ guide camera and found them to be undetectable. Thus we run the scintillometer whenever NIRSPEC is being used on-sky, typically 4 days per calendar month.

Figure 8 shows examples of two consecutive nights of scintillometer turbulence measurements (2010 0326 and 27 HST start dates). Systematic variations of the dome turbulence are seen, invalid points have a value of zero. On both nights several astronomical science targets were observed.

\subsection{TCS drive and pos. error signals}

Telescope motions result in image jitter, this degrades telescope performance. Wind induced telescope motions are detected by the TCS and corrected for. How effective this correction is something of great importance to the design of the TMT TCS. Confirming the expected characteristics of wind induced telescope motions, the susceptibility of the telescope to these disturbances and how well the TCS mitigates these disturbances is crucial to the design of TMT. Previous experiments at Keck have shown that the low frequency component of the elevation drive torque is proportional to the wind pressure on the telescope top end. We are now gathering data to allow an investigation of the variable components of the wind field and the telescope motion in both axes. 

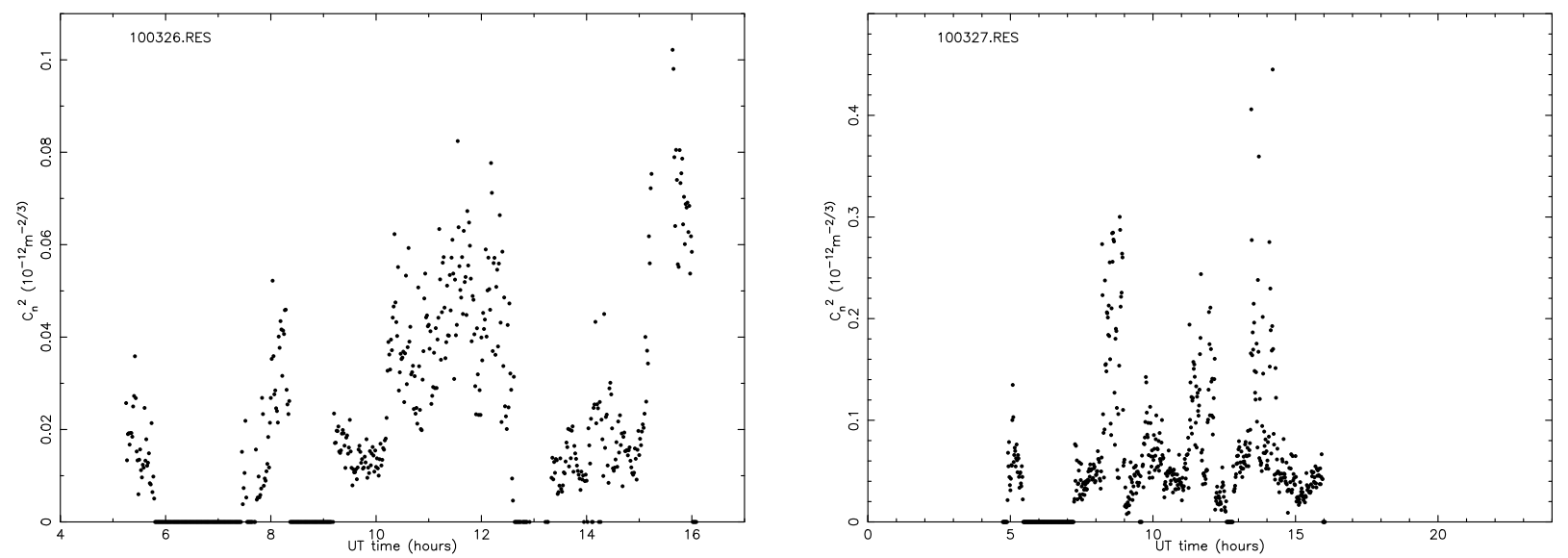

Figure 8. Examples of the scintillometer measurements of the optical turbulence in the Keck 2 dome. Left is from 2010 0326 (HST date at start of local night time), right is from 20100327.

Table 1. Log of experimental observations. Telescope log files that are routinely collected are not included here.

\begin{tabular}{l|l|l|l} 
& Start & End & Sampling \\
\hline Acoustic & 20080724 & 20080801 & $0.5 \mathrm{~Hz}-250 \mathrm{~Hz}, 3$ sensors \\
Vibration & 20080722 & 20080801 & $3 \mathrm{D}, 0.05$ to $250 \mathrm{~Hz}$, varied locations \\
Wind & 20090719 & - & $32 \mathrm{~Hz}, 3 \mathrm{D}, 2 \%, 5$ sensors \\
Temperature & 20090719 & - & $\sim 1 \mathrm{~Hz}, \pm 0.05^{\circ} \mathrm{C}, 5$ sensors \\
Dome turbulence & 201003 & Sporadic & $60 \mathrm{~s}$ \\
TCS drive & Development & - & $10 \mathrm{~Hz}$ \\
Log file data mining & NA & NA & -
\end{tabular}

We are measuring the Keck 2 TCS analog altitude and azimuth position error and drive torque signals at high frequency. The Keck TCS bandwidth is around $10 \mathrm{~Hz}$ and we are sampling at $200 \mathrm{~Hz}$ in order to capture all features. These measurements are continuously made using a simple ADC connected to the analog signals. Accurate time stamps allow correlation with the wind measurements described in Section 3.3.

\subsection{Status of wind, temperature, optical turbulence and TCS drive signal measurements}

Data gathering with the sensors installed on the telescope, dome top and the TCS signal acquisition system is all presently underway, see Table 1. No work has yet been done to begin correlating the dome seeing measurements with the wind and temperature fields. Similarly for the wind induced telescope motions, we plan to correlate the wind measurements against the TCS signal measurements. TCS signal measurements should begin in earnest in July 2010 after software updates to the DAS have been made. We are presently developing the data processing tools to generate the appropriate boundary conditions or inputs for the CFD and control system models and will process the measurements of the dome seeing and TCS signals appropriately to allow a comparison against the predictions of the models.

\section{UTILIZATION OF RESULTS}

The results and conclusions of the experimental work are described in TMT internal documents and data products are available to TMT design engineers. Humidity, vibration and acoustic measurements at Keck are incorporated into the TMT disturbance model which is being utilized extensively within the project. ${ }^{8}$

The vibration measurements and humidity characteristics at Keck have been instrumental in defining the primary mirror control system requirements. The Keck vibration environment featured strongly in the primary mirror segment actuator down select and guided the lab testing of actuators on the SSA. ${ }^{9}$ Edge sensor humidity response studies fed in strongly to edge sensor down select complemented with much lab testing. ${ }^{10}$ Measurements 
of vibrations and the humidity are feeding into instrument, secondary and tertiary mirror systems and telescope structure requirements documents.

\section{CONCLUSIONS}

Experimental efforts at Keck and involving archival log files from Keck are a work in progress. Some important lessons have been learned and applied and some information has been determined and gathered, namely:

- Disturbance model has been updated

- Vibration environment around the telescope pier

- Vibration propagation through the segment support assembly and of the M1 cell applied to actuator downselect

- Vibration properties of the the M1 surface

- Acoustic environment inside the Keck 1 enclosure suggests low risk for TMT

- Humidity environment quantified and effect on M1CS guides edge sensor selection

Work in progress includes the model validation efforts. Models are being developed for representative cases, input and comparison data is being acquired. Valuable experiences about engineering data collection are being learned through these efforts and being applied to the TMT engineering sensor system design. Further efforts to validate the CFD models involving the Canada France Hawaii Telescope are beginning at this time and previous efforts on the $4 \mathrm{~m}$ Blanco telescope also provide potentially useful experimental data. ${ }^{11}$

\section{ACKNOWLEDGMENTS}

The TMT project gratefully acknowledges the support of the TMT partner institutions. They are the Association of Canadian Universities for Research in Astronomy (ACURA), the California Institute of Technology and the University of California. This work was supported as well by the Gordon and Betty Moore Foundation, the Canada Foundation for Innovation, the Ontario Ministry of Research and Innovation, the National Research Council of Canada, the Natural Sciences and Engineering Research Council of Canada, the British Columbia Knowledge Development Fund, the Association of Universities for Research in Astronomy (AURA) and the U.S. National Science Foundation.

We acknowledge the support of the WMKO Operations and Infrastructure Department supervisors and technicians, Observing Support Department supervisors and observing assistants, Technical Services Department designers and Safety, Health and Environmental Affairs Officer.

\section{REFERENCES}

[1] Vogiatzis, K., et al. 2008, "Advances in aerothermal modeling for TMT", Proc. of the SPIE vol. 7017, p. 20

[2] Macmynowski, D., et al. 2008, "Dynamic analysis of TMT", Proc. of the SPIE vol. 7017, p. 26

[3] Cho, M., et al. 2010, "Thermal analysis of the TMT telescope structure", Proc. of the SPIE 7738-12, in press

[4] Macmynowski, D., et al. 2008, "Analysis of TMT primary mirror control-structure interaction", Proc. of the SPIE vol. 7012, p. 33

[5] Thompson, P., et al. 2008, "Control analysis of the TMT primary segment assembly", Proc. of the SPIE vol. 7017, p. 57

[6] Wetherell, E. 2006, "Accelerometer Hardware Description, V1.1", WMKO Internal Technical Document

[7] Vogiatzis, K., et al. 2010, "Thermal modeling environment for TMT", Proc. of the SPIE 7738-11, in press

[8] Macmynowski, D., et al. 2010, "Primary mirror dynamic disturbance models for TMT: vibration and wind", Proc. of the SPIE 7738-14, in press

[9] Regehr, M., et al. 2010, "Dynamic characterization of a prototype of the Thirty Meter Telescope primary segment assembly", Proc. of the SPIE 7733-85, in press

[10] Luong, B., et al. 2010, "Edge sensors for controlling segmented mirrors: a full industrial and turnkey product solution", Proc. of the SPIE 7733-82, in press

[11] Els, S., et al. 2010, "Monitoring of the environmental conditions inside the dome of the 4m Blanco Telescope at CTIO", Proc. of the SPIE 7733-141, in press 